\title{
NOTAS SOBRE ESPÉCIES DE IDIOPS PERTY, 1833 DO SUDESTE BRASILEIRO COM DESCRIÇÃO DE I. PIRASSUNUNGUENSIS SP. N. (MYGALOMORPHAE, IDIOPIDAE, IDIOPINAE)
}

\author{
Celina Yukari Fukami \& Sylvia Marlene Lucas \\ Biota Neotropica v5 (n1a)-http://www.biotaneotropica.org.br/v5n1a/pt/abstract?taxonomic-review+bn018051a2005
}

Recebido em 07/12/2003

Publicado em 01/02/2005

Instituto Butantan, Laboratório de Artrópodes Peçonhentos, Av: Vital Brazil 1500 CEP 05503-900, São Paulo, SP, Brasil. E-mail: celinayukari@butantan.gov.br \& sylvialucas@butantan.gov.br.

\begin{abstract}
The study of type and non-type material of species of the genus Idiops Perty, 1833, Southeastern Brazil, enabled to enhance the taxonomical knowledge of these species. Thus, Idiops montealegrensis Soares, 1944 is synonymized with Idiops camelus Mello Leitão, 1937; Idiops germaini Simon, 1892 described from Rio de Janeiro, Brazil, is confirmed as a valid species and Idiops pirassununguensis n. sp. is described from Pirassununga, São Paulo, Brazil. In addition, Idiops nilopolensis Mello Leitão, 1923, from Nilópolis, Rio de Janeiro is considered species inquirenda and new records are presented for Idiops camelus.
\end{abstract}

Key words: Idiopidae, Idiopinae, Idiops, new species, Southeastern of Brazil.

\section{Resumo}

O estudo de material tipo e não tipo do gênero Idiops Perty, 1833 das quatro espécies descritas para o sudeste do Brasil permitiu ampliar o conhecimento taxonômico das mesmas. Assim Idiops montealegrensis Soares, 1944 é sinonimizada com Idiops camelus Mello Leitão, 1937; Idiops germaini Simon, 1892, descrita para o Rio de Janeiro, é confirmada como espécie válida e Idiops pirassununguensis sp. n. é descrita para Pirassununga, São Paulo. Idiops nilopolensis Mello Leitão, 1923, de Nilópolis, Rio de Janeiro, cujo tipo não foi localizado, é considerada species inquirenda. São apresentadas novas ocorrências para Idiops camelus.

Palavras-chave: Idiopidae, Idiopinae, Idiops, espécie nova, sudeste do Brasil.

http://www.biotaneotropica.org.br 


\section{Introdução}

A família Idiopidae Simon, 1889 compreende três subfamílias: Arbanitinae Simon, 1903, Idiopinae Simon, 1899 e Genysinae Simon, 1903. Apenas dois gêneros ocorrem na América do Sul, Neocteniza Pocock, 1895 (Genysinae) com 11 espécies descritas e uma para o Brasil (Platnick 2004; Fukami et al. 2004) e Idiops Perty, 1833 (Idiopinae) com 17 espécies descritas, nove para o Brasil. Para a região sudeste do Brasil foram descritas quatro espécies: I. germaini Simon, 1892, I. nilopolensis Mello Leitão, 1923, I. camelus Mello Leitão, 1937 e I. montealegrensis Soares, 1944.

Nas coleções brasileiras, exemplares de Idiops são pouco representados e isto pode ser explicado por seus hábitos. As fêmeas e jovens são sedentários vivendo em túneis forrados de seda, cavados na terra (Capocasale 1972) ou junto aos troncos de árvores, fechando a entrada com um opérculo delgado, de difícil visualização (Pickard-Cambridge 1889). Os machos adultos são errantes sendo capturados com maior facilidade principalmente em armadilhas de solo. Nas coletas realizadas durante o desenvolvimento do projeto "Biodiversidade de Aracnídeos e Miriápodes do Estado de São Paulo” do programa Biota/Fapesp, foram capturados vários exemplares, que foram comparados com outros de diversas coleções brasileiras e com os tipos das espécies descritas para o sudeste brasileiro. Neste trabalho apresentamos algumas informações sobre as espécies, citamos novos registros para I. camelus Mello Leitão, 1937, estabelecemos uma sinonímia e descrevemos uma espécie nova para o Brasil. Quanto à espécie I. nilopolensis Mello Leitão, 1923, tipo não localizado, há necessidade de coleta de mais exemplares para confirmar a sua validade.

\section{Material e Métodos}

O material examinado pertence as seguintes instituições: IBSP - Instituto Butantan, São Paulo (A.D. Brescovit); MNHN - Museu de História Natural de Paris (C. Rollard); MNRJ Museu Nacional do Rio de Janeiro (A. B. Kury); MZSP-Museu de Zoologia, Universidade de São Paulo (R. Pinto da Rocha) e UNB - Universidade de Brasília (P. C. Motta).

Abreviaturas dos olhos: OLA, olhos laterais anteriores, OLP, olhos laterais posteriores, OMA, olhos médios anteriores, OMP, olhos médios posteriores. Todas as medidas são em milímetros. A genitália da fêmea foi submergida em óleo de cravo para estudo das estruturas internas. Os desenhos foram elaborados em estereomicroscopio Leica acoplado a uma câmara clara. As fotos em microscopia eletrônica foram feitas em microscópio eletrônico de varredura JEOL (JSM 840A) do Laboratório de Microscopia Eletrônica do Departamento de Física Geral do Instituto de Física da Universidade de São Paulo (USP).

\section{Taxonomia}

\subsection{Idiops pirassununguensis sp. $\mathbf{n}$.}

(Figs 1-5)

Tipo. Holótipo macho de Pirassununga, São Paulo, Brasil. X.1997, P. Valdujo col., depositado no IBSP 9565.

Etimologia. O nome específico refere-se à localidade onde foi coletado o holótipo, Pirassununga, São Paulo, Brasil.

Diagnose: Idiops pirassununguensis sp. n. aproximase de $I$. camelus por apresentar a apófise tibial da perna I dupla e grande, mas se distingue pelo aspecto do êmbolo longo e largo com uma dilatação na ponta (Figs. 1-2;5), pela presença de um pequeno tubérculo na face lateral interna do metatarso I (Fig. 3) e pelo maior número de dentes em todas as unhas superiores (Fig. 4). Distingue-se de $I$. germaini pois esta apresenta uma apófise tibial muito reduzida (Fig. 10) e não possui tubérculo no metatarso I.

Descrição. Macho (Holótipo). Comprimento total: 14,9. Carapaça: comprimento 7,2, largura 7,2. Lábio: comprimento 0,9, largura 1,3. Maxila: comprimento 2,7, largura 1,6. Olhos: OMA 0,36, OLA 0,46, OMP 0,24, OLP 0,46, OMA-OMA 0,20, OMA-OLA 0,74, OMP-OMP 0,42, OMP-OLP 0,18, OLA-OLP 0,92. Esterno: comprimento 3,8, largura 3,7. Pernas I - Fêmur 7,5/ patela 3,8/ tíbia 5,4/ metatarso 5,6/ tarso 2,4/ total 24,7, II - 6,5/ 3,2/ 4,3/ 4,6/ 2,0/ 20,6, III - 5,0/ 3,0/ 3,1/ 4,6/ 2,5/ 18,2, IV - 7,0/ 3,5/ 5,5/ 5,8/ 2,9/ 24,7. Palpo: 4,6/ 2,4/ 4,4/ 1,8/ 13,2. Coloração: dorso do corpo e pernas castanho escuro; ventre: esterno, lábio, maxilas, abdômen, coxas e trocanteres de colorido mais claro. Região cefálica pouco elevada com cerdas curtas e delicadas em pares, após o cômoro ocular, voltadas para trás e uma única cerda na frente dos OLA; fóvea torácica procurva; rastelo fraco numa pequena elevação, com algumas cerdas longas e finas; quelícera com 6 dentes grandes na promargem e alguns pequenos na retromargem; intumescência interqueliceral pequena, um pouco mais clara, bem próxima ao lábio coberta por uma mancha de pêlos escuros; tíbia do palpo com uma coroa de espinhos (Figs. 1-2,5); bulbo com êmbolo longo e achatado com uma dilatação apical (Figs. 1-2; 5); metatarso I com um pequeno tubérculo na face lateral interna (Fig. 3); apófise tibial dupla com ramo superior maior que o inferior (Fig. 3); unhas superiores I com 3 a 10 dentes, uniformes (Fig. 4).

Fêmea. Desconhecida

Distribuição. Brasil: Sergipe, Distrito Federal, Minas Gerais e São Paulo.

Material examinado. Brasil. Sergipe: Canindé de São Francisco, Usina Hidrelétrica Xingó, 1ठํ, 03/X/00, L. Lanuzzi (IBSP 10183); Distrito Federal: Brasília, $1 \overbrace{}^{2}$, 11/09/01, P. C. Motta (UNB 1276); Minas Gerais: Belo Horizonte, 1ठ, 20/IX/95, A. J. Santos (IBSP 9583), Paraopeba, $1 \delta$, VIII-IX/94, M. O. Gonzaga (IBSP 10178); 

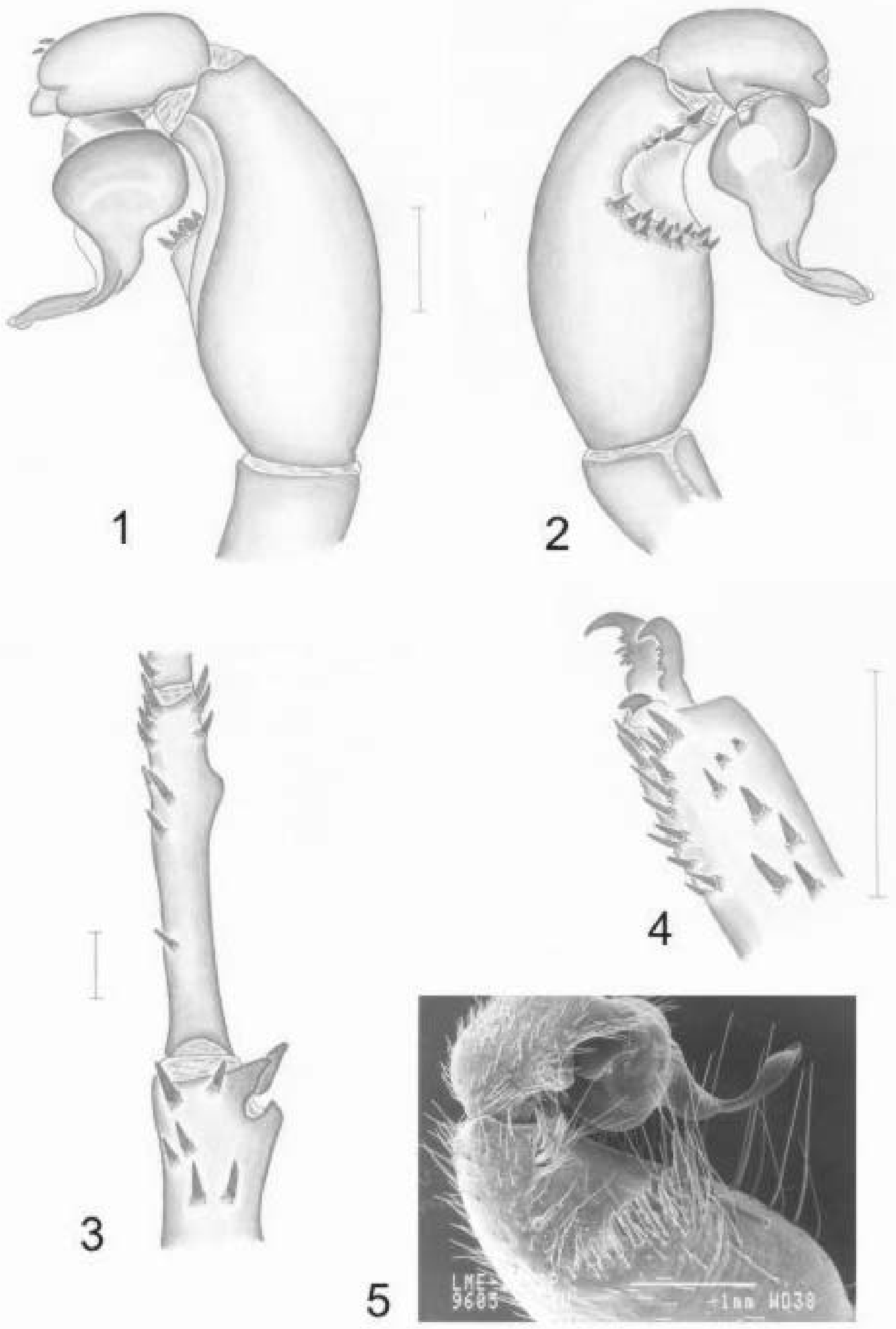

Figuras 1-5. Macho. Idiops pirassununguensis sp. n. 1. Palpo direito, prolateral; 2. Retrolateral; 3. Apófise tibial direita; 4. Garras do tarso esquerdo; 5. Foto de microscopia eletrônica, palpo direito retrolateral (Escala 1mm). 

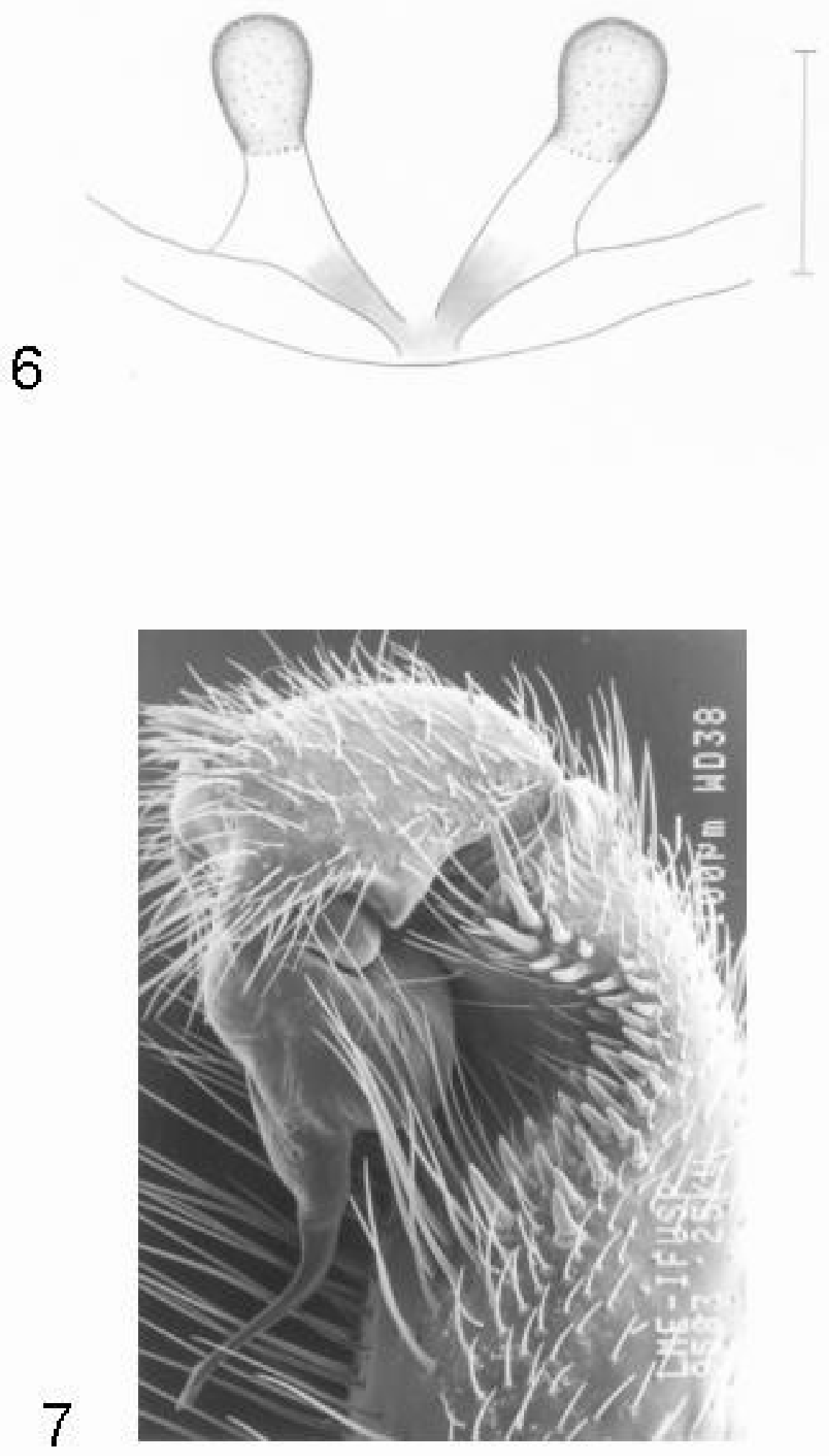

Figuras 6 e 7. Fêmea e macho. Idiops camelus. 6. Espermateca (Escala 1mm); 7. Foto microscopia eletrônica, palpo esquerdo, retrolateral (Escala $0,1 \mathrm{~mm}$ ). 

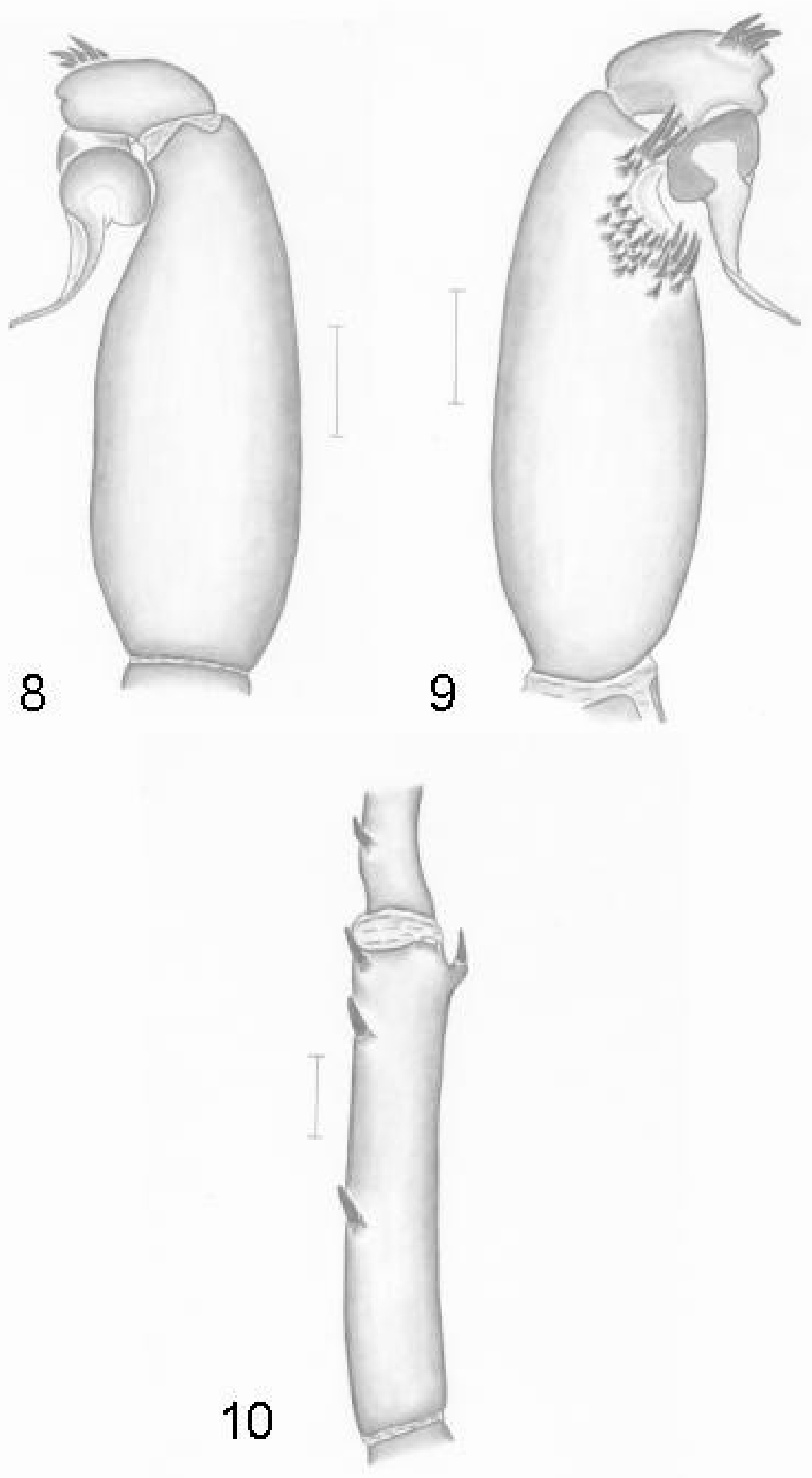

Figuras 8-10. Macho. Idiops germaini. 8. Palpo direito, prolateral; 9. Retrolateral, 10. Apófise tibial direita (Escala 1mm). 
São Paulo: Araraquara, 1ర̋, 30/IX/88 (IBSP 9562), São

Roque, $1 \delta^{2}, 02 / V I I I / 01$, A. B. Canute (IBSP 9133).

\subsection{Idiops camelus (Mello Leitão), 1937}

(Figs 6-7)

Pseudidiops camelus Mello Leitão, 1937: 1, fig. 1-2 (Holótipo fêmea de Corumbataí, São Paulo, Brasil, 1937, sem coletor, depositado no IBSP 3429, examinado).

Idiops camelus (Mello Leitão), 1937; Raven 1985: 158; Platnick 2004.

Idiops montealegrensis Soares, 1944: 156, figs. 2-4 (Holótipo macho da Fazenda Santa Maria, Monte Alegre, Amparo, São Paulo, Brasil, XI/1942, F. Lane col., depositado no MZSP E.485 C.326, examinado); Bücherl, 1957: 383, f. 22a; Platnick, 2004. SINÔNIMO NOVO.

Diagnose. Idiops camelus é uma espécie muito próxima de I. germaini pelo aspecto do bulbo copulador (Fig. 7) e pela ausência de um pequeno tubérculo na base do metatarso I; distingue-se desta principalmente pelo aspecto da apófise tibial que é maior; e de I. pirassununguensis sp.n. pois esta apresenta um tubérculo no metatarso I e o bulbo copulador tem um êmbolo mais largo com dilatação apical. A fêmea apresenta os receptáculos seminais simples com dilatação no ápice (Fig. 6).

Discussão. O estudo dos tipos e demais material das quatro espécies de Idiops descritas para a região sudeste do Brasil, permitiu concluir que I. montealegrensis é sinônimo de I. camelus. Apresentam o mesmo colorido, bem como o aspecto da carapaça, em forma de corcova de camelo, que Mello Leitão considerou a principal característica de $I$. camelus. Algumas diferenças como, por exemplo, presença de cúspides no lábio e maxila das fêmeas, ausêntes nos machos; e rastelo forte nas fêmeas e fraco nos machos, se devem ao dimorfismo sexual muito acentuado nas espécies deste gênero, como foi observado por Schiapelli \& Gerschman de Pikelin (1971).

Distribuição. Brasil: Espírito Santo, Minas Gerais, Rio de Janeiro, Santa Catarina e São Paulo.

Novos registros. Espírito Santo: São Mateus (Reserva Florestal da Companhia Vale do Rio Doce), 1 ㅇ 1ठో 2J, VII/1997, A. D. Brescovit (IBSP 7986; 9586; 9587);

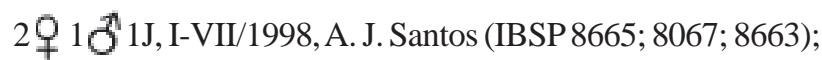
Minas Gerais: Alto Caparaó, Parque Nacional do Caparaó, 3ठో, V/2002, Equipe Biota (IBSP 10186; 10187; 10188); Ouro Preto, 19 (MNRJ 13631); Poços de Caldas, $1 \delta^{\Uparrow}$ (MNRJ 3456); Rio de Janeiro: Itatiaia, 19 (MNRJ 08); Petrópolis, 1 (MNRJ 14); Pinheiral (Fazenda Santa Helena), $8{ }^{\star} 1$ 1 1J, 5-11/XI/99, A. D. Brescovit (IBSP 10191-10198); São Paulo: São Paulo (Butantã), 1ठ, 20/X/88, A. Hoje (IBSP
9563); (Campus da Universidade de São Paulo, IPEN), 1B\&, 12/VIII/1991, P. J. Spencer (IBSP 9576); (Jardim Botânico), 1B\& (MZSP 7500); (Lapa), 1 9, 20/IV/1999, A. H. Bugelli (IBSP 9580); (Morumbi), 1ठ, 20/X/1968, O. Mamini (IBSP 293); (Parque Estadual da Cantareira), 2O , 18/VIII/1999, F. L. Silva (IBSP 7913; 9579); 66ð̋ 1ㅇ, IV-V/2000, S. Favorito et al leg (MZSP 21303-21309; 21311; 21315; 21317; 21320; 21328-21331; 21333; 21337; 21341; 21348); (Rio Pequeno), 1우 (IBSP 3346); (Rodovia Raposo Tavares, Km21), 1우, 21/ VII/1977, P. Alicke (IBSP 4270); Itariri (Ana Dias), 1q, M. Yamashita (IBSP 3364); Araçariguama, 19, VIII/1975, (IBSP 3977A); Caieiras, 1q, 17/XII/1965, P. J. César (IBSP 3749);

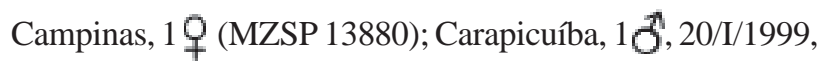
R. A. da Silva (IBSP 9577); Cotia, 1ठో, 11/IX/1996, J. P. A. Alves (IBSP 9561); Itirapina (Estação Ecológica de Itirapina), 1ㅇ 22/V/1999, M. E. Oliveira (IBSP 8789); 4ð”, VII-X/2001, G. Machado (IBSP 10184; 10185); Jarinú, 1ठ̋', 04/VIII/1989, J. Fitipaldi (IBSP 9572); Jundiaí, 1ㅇ , 30/V/1956, G. Righi (IBSP 3477); Limeira, 1ठ, 30/VIII/1990, A. M. Fernandes (IBSP 9575); Mogi das Cruzes (Parque Natural Municipal da Serra do Itapety), $10 ð$, V/2003, P. A. M. Goldoni (IBSP 10189; 10190); São Roque, 1 Q , 08///1999, J. C. C. Konte (IBSP 9578); Tapiraí, $1{ }^{0} 1$ 1, I/1999, C. A. Falcetti (IBSP 8257; 9581); Tietê 1O , 01/XII/1959, R. Seminário (IBSP 3521); Santa Catarina: Chapecó (Monte Alegre, $\left.27^{\circ} \mathrm{S} 50^{\circ} \mathrm{W}\right), 6{ }^{\circ}$, 22/IV/ 20002, J. M. M(IBSP 10199; 10200; 10201).

\subsection{Idiops germaini Simon, 1892}

(Figs 8-10)

Idiops germaini Simon, 1892: 92. (Holótipo macho do Rio de Janeiro, Brasil, 1892, Germain col., depositado no MNHN AR 4174, examinado); Platnick, 2004.

Diagnose. I. germaini aproxima-se de I. camelus pelo aspecto do bulbo copulador com êmbolo longo e ponta afilada, porém mais comprido (Figs. 8-9); distingue-se de $I$. camelus e de I. pirassununguensis sp. n. pela apófise tibial bem reduzida (Fig. 10).

Discussão. Mello Leitão (1923) redescreveu a espécie baseada em exemplares coletados em Mendes, Rio de Janeiro, machos e fêmeas, citando "tíbias do primeiro par de pernas cilíndricas, armadas de duas pequenas apófises, apicais, subiguais na borda interna.” Não vimos este material e os exemplares examinados do Rio de Janeiro não apresentaram estas características.

Distribuição. Brasil: Rio de Janeiro

Material examinado. Apenas o holótipo. 


\subsection{Idiops nilopolensis Mello Leitão, 1923}

Idiops nilopolensis Mello Leitão, 1923: 47 (Holótipo fêmea de Nilópolis, Rio de Janeiro, Brasil, 1923, Blanc de Freitas col., deveria estar depositado no MNRJ, não localizado, provavelmente perdido); Bücherl, Timotheo \& Lucas, 1971: 121, figs. 6-7; Platnick, 2004.

Discussão. Não foi possível a identificação precisa da espécie pela descrição original, muito resumida e, que omite caracteres importantes como a forma das espermatecas. Bücherl et al (1971) examinaram o tipo, apresentaram as medidas do esterno e citam que as garras superiores têm um dente submediano grande e outro abaixo bem menor, há um desenho esquemático das espermatecas, dois lobos com dilatação apical. Consideraram a espécie válida. Não localizamos o tipo, as espermatecas são semelhantes às de I. camelus. Até que se possa obter material da localidade-tipo, para uma melhor avaliação, consideramos a espécie I. nilopolensis como species inquirenda.

\section{Agradecimentos}

Aos curadores pelo empréstimo do material; aos Prof. Pedro Kiyohara e a técnica Simone Perche de Toledo (USP) pelas fotos de microscopia eletrônica; aos Drs. Antonio. D. Brescovit e Fernando Pérez-Miles pela leitura crítica do manuscrito. O trabalho foi financiado pela Fundação de Amparo à Pesquisa do Estado de São Paulo (FAPESP processos 99/ 05446-8 (Biota/Fapesp) e 01/14368-2 (primeira autora).

\section{Referências Bibliográficas}

BÜCHERL, W., TIMOTHEO DA COSTA, A. \& LUCAS, S. M. 1971. Revisão de alguns tipos de aranhas caranguejeiras (Orthognatha) estabelecidos por Cândido de Mello-Leitão e depositados no Museu Nacional do Rio. Mem. Inst. Butantan 35: 117-138.

CAPOCASALE R. 1972. Observaciones eco-etologicas sobre Idiops clarus (Mello-Leitão) (Araneae; Ctenizidae). Com. Zool. Mus. Montevideo. 136: 1-15.

FUKAMI, C. Y., LUCAS, S. M. \& INDICATTI, R. P. 2004. Primeiro registro do gênero Neocteniza Pocock, 1895 para o Brasil (Araneae, Mygalomorphae, Idiopidae, Genysinae). Revta. Ibérica Aracnol., 9: 30.

MELLO-LEITÃO, C. F. DE. 1923. Theraphosideas do Brasil. Rev. Mus. Paulista 13: 1-438.

PICKARD-CAMBRIDGE, O., 1889. On a new tree trap-door spider from Brazil. Proc. zool. Soc. Lond. 1889: 250-252.

PLATNICK, N. I. 2004. The world spider catalog, version 5.0. American Museum of Natural History, online at http:/ /research.amnh.org/entomology/spiders/catalog/ index.html. (Acessado em IX/2004).

RAVEN, R. J. 1985. The spider infraorder Mygalomorphae (Araneae): Cladistics and systematics. Bull. Am. Mus. nat. Hist. 182: 1-180.

SCHIAPELLI, R. D. \& GERSCHMANDE PIKELIN., B. S. 1971. Estudio de algunas arañas descriptas por Mello-Leitão para el Uruguay. Revta Soc. ent. argent. 33: 57-62.
Título: Notas sobre o gênero Idiops Perty, 1833 do sudeste brasileiro com a descrição de $I$. pirassununguensis sp. n. (Mygalomorphae, Idiopidae, Idiopinae).

Autores: Celina Yukari Fukami \& Sylvia Marlene Lucas

Biota Neotropica, Vol. 5 ( número 1a): 2005

http://www.biotaneotropica.org.br/v5n1a/pt/ abstract?taxonomic-review+bn018051a2005

Recebido em 07/12/2003 - Publicado em 01/02/2005

ISSN 1676-0603 